\title{
Multiple-Criteria Cost Analysis for Simulated Life Cycle of Office Building
}

\author{
Michal Krzemiński
}

\author{
Department of Production Engineering and Construction Management, Institute of Building \\ Engineering, Faculty of Civil Engineering, Warsaw University of Technology, \\ Al. Armii Ludowej 16,00-637 Warsaw, Poland, m.krzeminski@il.pw.edu.pl
}

\begin{abstract}
The article presents an office building life-cycle estimating possibility with the use of multiple-criteria evaluation and simulations modeling techniques. The first part describes the life cycle of an office building with a focus on the places where it is necessary to make strategic decisions. These decisions are most often associated with (the incurring of) high financial costs. It is, therefore, necessary to choose a tool supporting the decision-making process. Presents for evaluation several criteria were developed, a number of which concern office buildings. It should be remembered that the multiple-criteria assessment means not only the criteria but also weights which are assigned to them. The article discusses different ways of assigning weights to criteria, e.g. the Simos method. The use of several different methods of multiple-criteria evaluation was suggested, for example the method of weighted sum, the ideal point method and the entropy method. The analysis was based on simulation models. The full cost model was built in MS Excel. For modeling the Crystal Ball software was used. The software applied allowed not only to determine confidence in the selected scenarios, but also to point to the criterion or factor which has the greatest impact on the final result. Such information can be obtained through the use of sensitivity analysis.
\end{abstract}

Keywords: Office Building, Life-Cycle, Multiple-Criteria Analysis, Crystal Ball.

\section{Introduction}

The definition of the life cycle of an office building can be fully based on the definition of the life cycle of a building as such. According to the definitions in ISO 15686-5: 2008, the life cycle of a building can be described as successive, interrelated periods of time between the selected time and the time of withdrawal or disposal of the building, in which certain criteria are assessed (e.g. costs, energy consumption, etc.).This period can be specified in the analysis, e.g. as a period equal to the time of renting or owning, or may cover the entire life cycle of a building, while the life cycle period is subject to the definition of the scope and specificity of operational requirements for a given building component. The definition includes a reference to the criteria of assessing the life cycle of an existing building. In the article, the author approaches the topic through the prism of strategic decisions accompanying the entire period in which an office building project is considered. These decisions are made throughout the entire life cycle of the project, at the initial, design, implementation and operational stages.

\section{Strategic Decision Points}

The most important points of strategic decisions, according to the author, are listed below. They have also been briefly characterized.

- Location Selection - different types of places: best, good, average, cheap, dedicated office buildings (e.g. near/in logistics centers ...). 
- Widely understood construction technology that often affects the initial cost of the object. The cost is associated with the standard, which in many cases is the result of the location in which the object is designed.

- Permanent/Short-term tenants. There are various office buildings. In many cases, the assumption is that they are built for long-term tenants who are also strategic tenants and who often attract smaller ones. However, there are more and more advertisements of objects in which space is sublet for short periods of time, sometimes even for days.

- Renovations/Modernizations. The aims of renovation / modernization are an important issue. The existence of an office object is only justifiable when it brings profit. It is known that in time a building ceases to be as attractive as it was at the beginning. The aim of renovation, as it is understood by the author, is mainly to maintain the standard and output functionality. The aim of modernization, however, is to change the object in a way which makes it possible to place it at the top of the list of attractive objects in a given environment, which is its reference environment.

\section{Renovations/Modernizations}

According to the author, the scope of renovation and modernization works can be divided into four basic types, which are listed below. A set of criteria was also developed to evaluate these variants.

basic renovation - aimed only at maintaining the object in working condition,
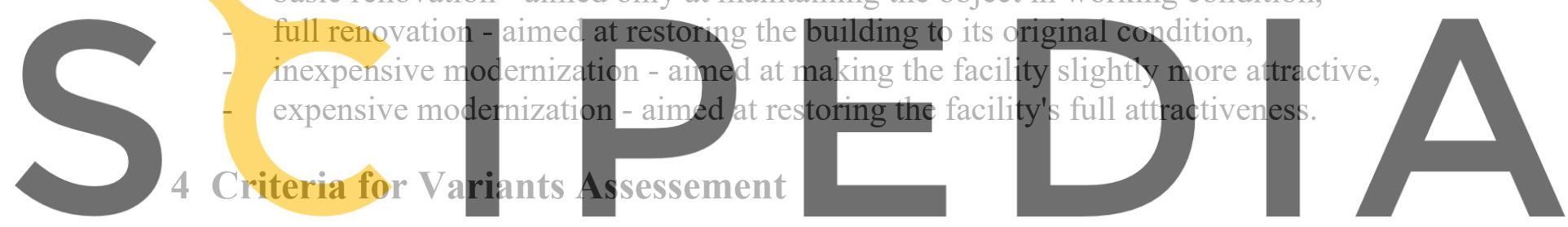

The set of criteria includes four elements. The first three measurable criteria, which do not

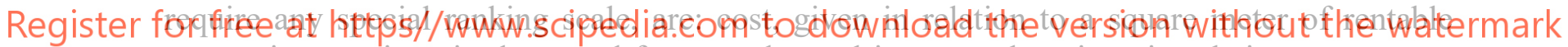
space, time - given in days, and forecasted rental income, also given in relation to a square meter of rentable space. For the last criterion, which is the scope of works, a four-point scale was adopted. A description of the scale is presented below. It should be noted here that the increasement of scope may result in entering more and more serious construction works. With proper planning, the permit obtaining process and the design process should not pose a problem, but the construction process itself may already be problematic as unplanned delays, like on any construction site, may occur.

- 1 - the smallest scope of renovation and modernization works understood as the exchange of those elements only which are necessary to be replaced in order to meet the requirements, more technical than aesthetic. In this case, there is rather no risk of delays.

- 2 - is the scope of renovation which is characterized by incurring the expenditure necessary to restore the object to the condition of commissioning. It assumes greater work effort than in the first case as well as the probability of greater costs generated by the need to replace elements that admittedly meet the technical requirements but no longer meet the aesthetic requirements. Sometimes in the case of, e.g. facades, this can already be a high cost within the meaning of a significant range. Slight delays are 
possible here.

- 3 - this note corresponds to renovation / modernization in which, apart from bringing the object to its full technical and aesthetic usability, minor changes are also made to the object. Such changes are, for example, a slight reconstruction of the facade including the modernization of the entrance portal. Changes can also be carried out inside the building and may involve, for example, the rearrangement of partition walls. In this case there is a risk of delay, which in extreme situations may become perceptible.

- 4 - such a note should be attributed to the renovation / modernization, which in principle will carry a significant scope of construction works. It may include works related to the complete reconstruction of the façade or to the modernization of communication routes, e.g. the addition of subsequent elevators. Such renovation/modernization can be considered as a reconstruction of the facility which carries a significant risk of delays.

\section{Weight Scenarios, Assigning Weights Using the Simos Method}

The original Simos method is based on the following three steps. First step is when the Decision Maker (DM) gives a set of cards with the name of one criterion the names of various criateria and a set of white cards. The number of white cards depends on the DM. In the next step the DM is asked to rank the cards from the most to the least important. If two cards are of

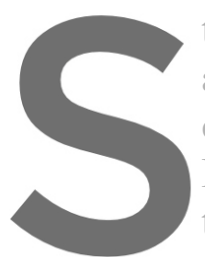
the same importance they are placed between the cards are placed betwee

B. Basing on this we cat the rank. Next, the distance between criteria is
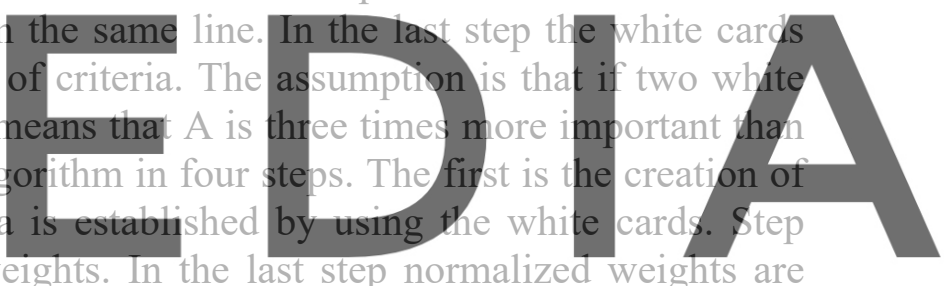

three is the calculation of non-normalized weights. In the last step normalized weights are

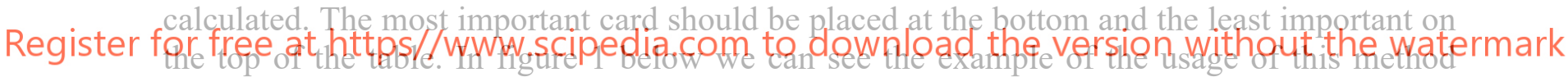
based on previously described criteria.

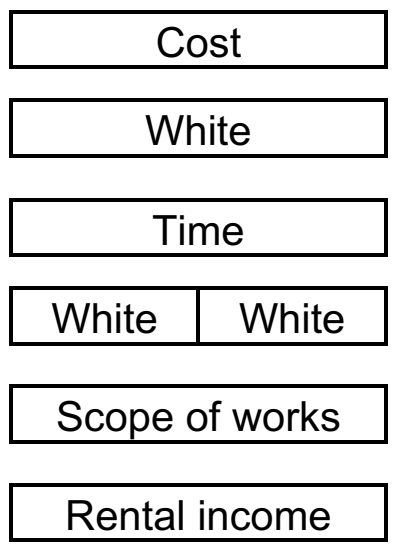

Figure 1. Cards order according to Simos method.

Table 1 below shows the results of calculations done with the use of Simos method. 
Table 1. Criteria weight calculations with the use of Simos method.

\begin{tabular}{lcccc}
\hline & $\begin{array}{c}\text { Cards } \\
\text { number }\end{array}$ & Position & $\begin{array}{c}\text { Non-normalized } \\
\text { Weights }\end{array}$ & Normalized weights \\
\hline Cost & 1 & 1 & 1 & 0,06 \\
\hline White card & 1 & 2 & $\ldots$ & $\ldots$ \\
\hline Time & 1 & 3 & 3 & 0,18 \\
\hline White card & 2 & $4 ; 5$ & $\ldots$ & $\ldots$ \\
\hline Scope of works & 1 & 6 & 6 & 0,35 \\
\hline Rental income & 1 & 7 & 7 & 0,41 \\
\hline
\end{tabular}

In the fourth line of the table we can find two white cards. The author's assumption was that these two cards make bigger span between the criteria.

\section{Multi-Criteria Assessment Methods}

Weighted sum and ideal point methods are among the most basic methods of multi-criteria assessment. They are widely known and, according to the author, there is no need to quote their definitions and descriptions. The entropy method described below is more interesting.

The entropy is a thermodynamic parameter of the state of a system. However, in the statistical interpretation, it determines the degree of not ordering the harvest (or the degree of uniqueness). According to the Polish language dictionary, entropy in thermodynamics means

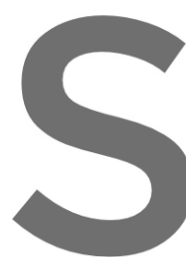
the amount equal to th reversible process. In in disorderness. The Entropy mathod allows us describing the options in question based information was first applied by Claude Sha

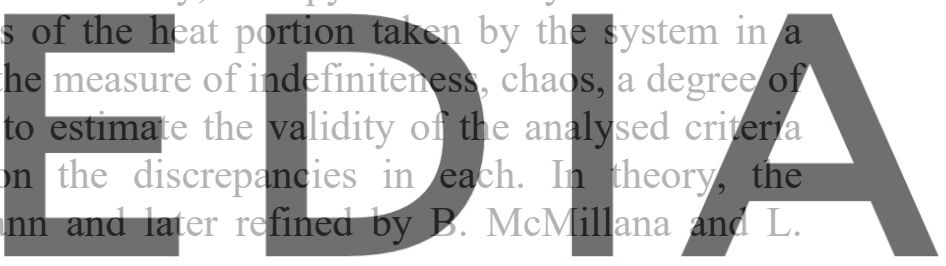
Breiman. Based formula in entropy method is:

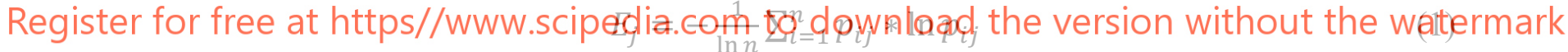
where:

$\mathrm{j}$ - number of criterion,

$\mathrm{i}$ - number of assessed variant (from 1 to $\mathrm{n}$ ),

p - assessment value.

It is easy to notice that if all the criteria have the same value of assessment, the entropy equals 1. For weights vector modification we use volatility level $\mathrm{z}$ based on following formula:

$$
z_{j}=1-E_{j}
$$

Criteria weights vector is modified by using:

$$
w_{j}^{0}=\frac{\frac{z_{j}}{\sum_{j=1}^{m} z_{j}} * \bar{w}_{j}}{\sum_{j=1}^{m}\left(\frac{z_{j}}{\sum_{j=1}^{m} z_{j}} * \bar{w}_{j}\right)}
$$

where: $\overline{\mathrm{w}}_{\mathrm{j}}-$ weights established by DM, $\mathrm{w}_{\mathrm{j}}^{0}-$ new weights. 


\section{Example}

Analyzed example concerns medium class office building located in Warsaw in range of $5 \mathrm{~km}$ from Palace of Culture and Science. Unnecessary data was collected in table below.

Table 2. Assessed investment options matrix.

\begin{tabular}{lccc}
\hline & Variant 1 & Variant 2 & Variant 3 \\
\hline Cost & 180 & 210 & 270 \\
\hline Time & 15 & 40 & 60 \\
\hline Scope of works & 1 & 2 & 3 \\
\hline Rental income [monthly] & 50 & 55 & 75 \\
\hline
\end{tabular}

Standarized grades used for assessment are in below table number 3.

Table 3. Standardized grades matrix.

\begin{tabular}{lccc}
\hline & Variant 1 & Variant 2 & Variant 3 \\
\hline Cost & 0,47 & 0,54 & 0,70 \\
\hline Time & 0,20 & 0,54 & 0,81 \\
\hline Scope of works & 0,27 & 0,53 & 0,80 \\
\hline Rental income [monthly] & 0,66 & 0,60 & 0,44 \\
\hline
\end{tabular}

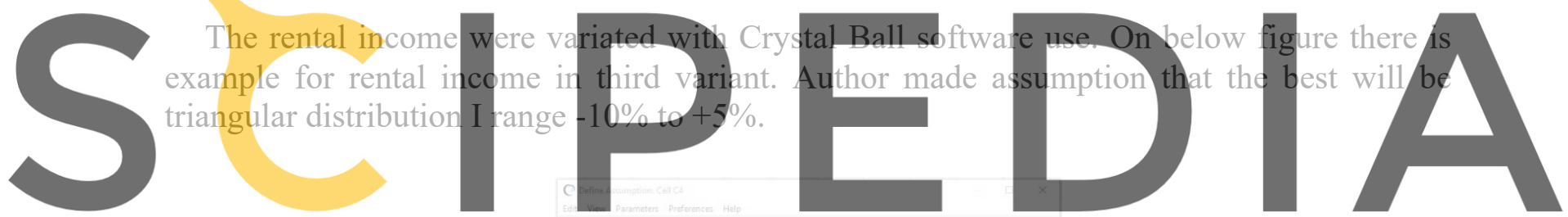

Register for free at https//www.scipedia.com to download the version without the watermark

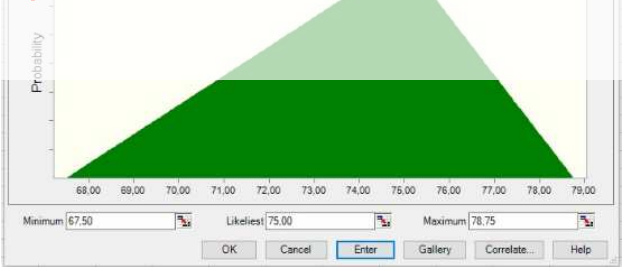

Figure 1. Triangular distribution for rental income variant 3

Calculation results were collect in below table 4. As it was previously mentioned calculation were made with three method use. Weighed sum, ideal point and entropy method. With accordance to all of this three method order was the same. The best was investment variant number one(minimum was looking for). Probably because cost of renovation and rental cost were going together proportionally, but the risk of delay grow quickly in meaning scope of works criterion. The result also shows how important this criterion actually is. 
Table 3. Standardized grades matrix.

\begin{tabular}{lccc}
\hline & Variant 1 & Variant 2 & Variant 3 \\
\hline Wighted sum & 0,43 & 0,56 & 0,65 \\
\hline Ideal point & 0,29 & 0,54 & 0,71 \\
\hline Entropy method & 0,17 & 0,34 & 0,48 \\
\hline
\end{tabular}

\section{Conclusions}

The article defines the life cycle of a building object together with the definition of strategic decision points. The assessment of the points regarding the decision on renovation or modernization was mainly considered.

A set of criteria have been developed to make the choice of the right option easier. In addition to the criteria, a weighting scenario was created with the use of Simos method. The entropy method used in the assessment was also described. During calculation, the expected rental income was established with the use of CrystalBall software.

The calculations made for the presented example show that this approach to the subject, in which various advanced methods are combined in multi-criteria assessment, gives good results and can be used assessing renovation and modernization options for office buildings.

\section{ORCIID}

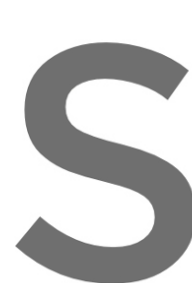

Michał Krzemiński: http:/orcid.org/0000-0002-6352-5942

References

Biruk, S., Jaśkowski, P. and Krzemi windows for their availability. Arch Figueiraa, J, and Roy, B. (
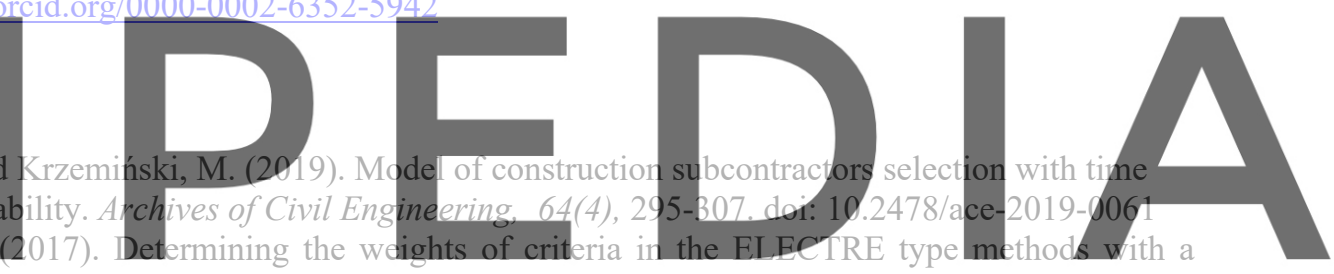
revised Simos' procedure. European Journal of Operational Research 139, 317-326

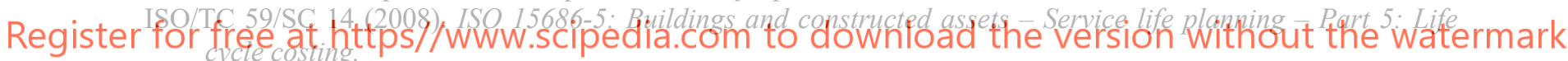

Juszczyk, M. and Leśniak, A. (2019). Modelling Construction Site Cost Index Based on Neural Network Ensembles. Symmetry-Basel 11(3), 411. doi:10.3390/sym11030411

Krzemiński, M. (2016). Comparison of selected multi-criteria assessment methods. AIP Conference Proceedings 1738, 200004. https://doi.org/10.1063/1.4951976

Krzemiński, M. (2016). Construction scheduling and stability of the resulting schedules. Archives of Civil Engineering, 57(2), 89-100. doi: J0.1515/ace-2015-0067

Nowak, K. (2019). The Elasticity of the Office Space Market in Poland: Looking for the London Effect. World of Real Estate Journal, 5-18. doi: 10.14659/WOREJ.2019.108.01

Olszewski, K., Decyk, P. and Gołaszewska, K., (2018). Hedonic analysis of office and retail rents in three major cities in Poland. Recent trends in the real estate market and its analysis, SGH Warsaw School of Economics 383-392.

Siskos, E. and Tsotsolas N. (2015). Elicitation of criteria importance weights through the Simos method: Arobustness concern. European Journal of Operational Research 246, 543-553.

Syed, A. I. H. and Mandal U. K. (2016). Entropy based MCDM approach for Selection of material. National Level Conference on Engineering Problems and Application of mathematics pp.1-7, Agartala, Tipura, India

Wieczorek, D., Plebankiewicz, E. and Zima, K. (2019). Model estimation of the whole life cost of a building with respect to risk factors. Technological and Economic Development of Economy 25(1), 20-38. doi.org/10.3846/tede.2019.7455 